TITLE:

\title{
Effects of magnetic curvature on the lower-hybrid-drift instability
}

\author{
$\operatorname{AUTHOR}(\mathrm{S})$ : \\ Ueno, $G$
}

\section{CITATION:}

Ueno, G. Effects of magnetic curvature on the lower-hybrid-drift instability. PHYSICS OF PLASMAS 2001, 8(4): 1159-1170

\section{ISSUE DATE:}

2001-04

URL:

http://hdl.handle.net/2433/49926

\section{RIGHT:}

Copyright 2001 American Institute of Physics. This article may be downloaded for personal use only. Any other use requires prior permission of the author and the American Institute of Physics. 


\title{
Effects of magnetic curvature on the lower-hybrid-drift instability
}

\author{
Genta Ueno \\ Department of Geophysics, Graduate School of Science, Kyoto University, Kitashirakawa-Oiwake-cho, \\ Sakyo, Kyoto 606-8502, Japan
}

(Received 24 August 2000; accepted 17 January 2001)

\begin{abstract}
A local dispersion relation is derived for the lower-hybrid-drift instability including the effects of magnetic curvature associated with transverse electromagnetic perturbations. To account for the curvature drift, an alternative method proposed by Nakamura [Phys. Plasmas 4, 3765 (1997)] is applied to obtain the perturbed distribution function. It is found that the previous treatment, i.e., simulating the curvature drift by a virtual gravitational drift, is considerably inaccurate. When an ambient magnetic field has a curvature so that the curvature drift is directed opposite the $\nabla B$ drift, the maximum growth rate increases as long as the radius of curvature is larger than a certain value, while the rate decreases for a sharper magnetic field curvature. At the same time, the wave number giving the maximum growth decreases monotonically. The growth rate is increased by a curvature drift coincident with the $\boldsymbol{\nabla} B$ drift. The effects of the magnetic curvature become larger in high-beta plasmas. (C) 2001 American Institute of Physics. [DOI: 10.1063/1.1354646]
\end{abstract}

\section{INTRODUCTION}

The lower-hybrid-drift instability is a high-frequency $\left(|\omega| \gg \Omega_{i}\right)$ drift-wave instability driven by cross-field currents in the presence of inhomogeneities in density $n(x)$ and magnetic field $B_{0}(x)$ [Fig. 1(a)]. Here, $\omega=\omega_{r}+i \omega_{i}$ is the complex eigenfrequency of the wave perturbation, $\Omega_{i}=|+e| B_{0} / m_{i}$ is the ion Larmor frequency, $e$ is the elementary electric charge, and $m_{i}$ is the ion mass. The characteristic growth rate $\omega_{i}$ and perpendicular wave number $k_{y}$ satisfy (assuming flute perturbations with $\mathbf{k} \cdot \mathbf{B}_{0}=0$ )

$$
\omega_{i} \approx \Omega_{L H}, \quad k_{y}^{2} r_{L e}^{2} \approx 1,
$$

for $T_{e} \approx T_{i}$ and $V_{E} / v_{i} \gtrsim 1$. Here, $\Omega_{L H}=\omega_{p i} /(1$ $\left.+\omega_{p e}^{2} / \Omega_{e}^{2}\right)^{1 / 2}$ is the lower-hybrid frequency, $r_{L e}=v_{e} / \Omega_{e}$ is the thermal electron Larmor radius, $T_{e}$ and $T_{i}$ are the electron and ion temperatures, $V_{E}$ is the $\mathbf{E} \times \mathbf{B}$ drift velocity, $v_{e}$ $=\sqrt{2 T_{e} / m_{e}}$ and $v_{i}=\sqrt{2 T_{i} / m_{i}}$ are the electron and ion thermal speeds, $\omega_{p e}=\sqrt{n e^{2} / \epsilon_{0} m_{e}}$ and $\omega_{p i}=\sqrt{n e^{2} / \epsilon_{0} m_{i}}$ are the electron and ion plasma frequencies, $\Omega_{e}=|-e| B_{0} / m_{e}$ is the electron Larmor frequency, and $m_{e}$ is the electron mass. Since the lower-hybrid-drift instability requires fairly narrow sheaths and is favored by $T_{e}<T_{i}$, it appears to arise in highdensity magnetic compression devices such as reversed field theta pinches ${ }^{1,2}$ and may also be found in a certain region of the Earth's magnetotail. ${ }^{3}$ Furthermore, recent space plasma observations with high resolution revealed the lower-hybrid wave near the magnetic reconnection site and enabled us to compare the observation with the theory in detail. ${ }^{4}$

When we construct the theory for the comparison, we should quantitatively account for the effects of finite beta and magnetic curvature. The former is because a high beta such as in the plasma sheet is known to act as a stabilizing factor for the lower-hybrid-drift instability. It is explained by the resonance of an electron $\boldsymbol{\nabla} B$ drift and the wave, ${ }^{5,6}$ and was treated comprehensively by Davidson et al. ${ }^{7}$ including both electromagnetic and $\nabla B$ drift effects.

On the other hand, the latter (magnetic curvature) brings an electron curvature drift which cancels the effect of the $\nabla B$ drift, so that the lower-hybrid-drift instability can be destabilized again. ${ }^{4}$ Actually, when we observe the nearEarth magnetotail, the normal field exists which gives curvature to the reversed magnetic field. The magnetic curvature was, however, treated insufficiently as explained below. Historically, Krall and McBride ${ }^{8}$ included the effect of magnetic field curvature in the theory of lower-hybrid-drift instability in order to use the theory in a detailed way to model transport, since real applications (e.g., tokamak) must include curvature effects. Accordingly, when taking account of the magnetic field curvature, they replace the centrifugal force on particles by a virtual gravitational force for simplicity. With this gravitational drift approximation, Shinohara et $a .^{4}{ }^{4}$ derived the dispersion relation of the lower-hybrid-drift instability in a finite plasma beta and examined whether the "curvature drift" (i.e., the virtual gravitational drift) can modify the electron $\nabla B$ drift effects.

For such a treatment of the curvature drift, however, Huba and Drake ${ }^{9}$ pointed out that wave-particle resonances cannot occur and only the nonresonant behavior of the particles due to the curvature drift is considered; they focused on curvature drift resonances while ignoring $\nabla B$ drift and noted that the electron energized through the curvature drift always leads to wave damping. Hence, the resonance mechanism by the curvature drift differs essentially from that by the virtual gravitational drift, but Huba and Drake ${ }^{9}$ merely indicated the qualitative importance of considering the curvature drift properly and did not derive the dispersion relation of the lower-hybrid-drift instability including the effects of the curvature drift. This is because there has been difficulty in treating curvature drifts which appear when a linear- 
(a) Straight Magnetic Field

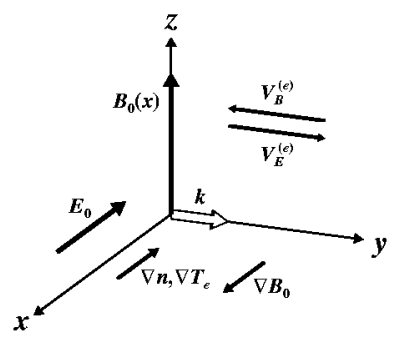

(b) Curved Magnetic Field $\left(\boldsymbol{R}_{c}>0\right)$

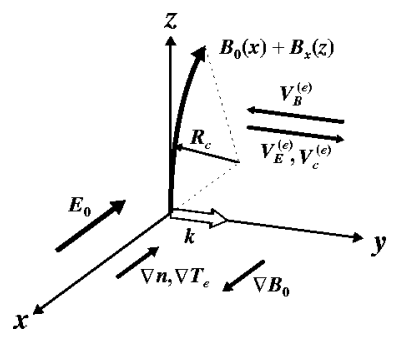

(c) Curved Magnetic Field $\left(\boldsymbol{R}_{c}<0\right)$

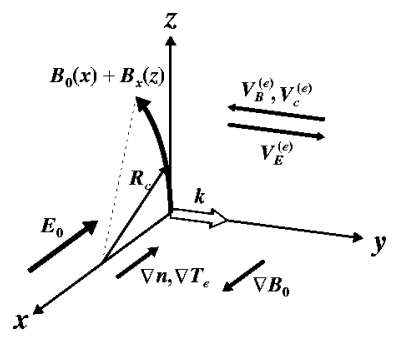

FIG. 1. Slab geometry and background plasma configuration: (a) straight magnetic field line, (b) curved magnetic field line with $R_{c}>0$, and (c) curved magnetic field line with $R_{c}<0$.

ized Vlasov equation is solved by the method of characteristics, also called "integration over unperturbed orbits." This method can give us a formal solution of the linearized Vlasov equation, but in practice, it is worth using only when we can describe the equilibrium distribution function in terms of the constants of motion: a differentiation of the equilibrium distribution function by a constant of motion is also a constant of motion, so the term can be factored out of the integral over the unperturbed orbit. In the presence of magnetic curvature, the method of characteristics does not work well due to the fluctuation of what was a constant of motion in the absence of curvature.

Recently, Nakamura ${ }^{10}$ proposed an alternative method for solving the linearized Vlasov equation. With this method, we can obtain the solution by combining the perturbed orbits and the differentiation of the equilibrium distribution function calculated separately, and can apply the problem to where the method of characteristics does not work in a practical sense. In particular, this alternative method enables us to treat curvature drifts properly and a dispersion relation has been derived for simple electrostatic perturbations by the lower-hybrid-drift instability including the case where both the $\nabla B$ drift and curvature drift are concurrent.

The purpose of this paper is to extend his work to a more realistic situation, that is, to derive and analyze a local dispersion relation for the lower-hybrid-drift instability including magnetic curvature effects associated with transverse electromagnetic perturbations. It is shown that the previous calculations, in which curvature drifts were simulated by virtual gravitational drifts, are considerably inaccurate; curvature drifts must be treated precisely as in the present paper. The organization of this paper is as follows. In Sec. II we derive perturbed distribution functions for the lower-hybriddrift instability with a curved magnetic field as well as for the traditional lower-hybrid-drift instability with a straight magnetic field by means of the alternative method. The local dispersion relation for the lower-hybrid-drift instability is obtained in Sec. III, including magnetic curvature effects with transverse electromagnetic perturbations. In Sec. IV, the local dispersion relation is solved numerically in the parameter regime of greatest interest for high density pinches, i.e., $T_{e}$ $\approx T_{i}$ and plasma betas on the order of unity. Finally, in Sec. $\mathrm{V}$, a summary of results and conclusions are presented.

\section{PERTURBED DISTRIBUTION FUNCTION}

The physical model and coordinate system in this paper are taken to be similar to those in earlier publications. ${ }^{2,3,7,11}$ The background plasma configuration and slab geometry employed in the present analysis are illustrated in Fig. 1. Figure 1(a) has a straight ambient magnetic field $\mathbf{B}_{0}(x)$ in the $+z$ direction, with an electric field $\mathbf{E}_{0}$ in the $-x$ direction, and density and temperature gradients $\left(\boldsymbol{\nabla} n\right.$ and $\left.\boldsymbol{\nabla} T_{e}\right)$ also in the $-x$ direction while the magnetic field gradient $\left(\nabla B_{0}\right)$ is in the $+x$ direction. This is a traditional simplified situation for the lower-hybrid-drift instability, in which $\mathbf{E} \times \mathbf{B}$ and $\boldsymbol{\nabla} B$ drifts and wave resonances occur. In this case, the electron drift motion consists of an $\mathbf{E} \times \mathbf{B}$ drift in the $+y$ direction and a $\nabla B$ drift in the $-y$ direction.

Now we consider the effect of magnetic field curvature by adding a new component of a magnetic field $\mathbf{B}_{x}(z)$ in the $-x$ or $+x$ directions as shown in Figs. 1(b) or 1(c). In Fig. 1(b), the total ambient magnetic field $\mathbf{B}_{0}(x)+\mathbf{B}_{x}(z)$ is curved with the radius of curvature $R_{c}$ directed to the $+x$ direction while the same with $R_{c}$ directed to the $-x$ direction in Fig. 1(c). Then, the curvature drift has the opposite direction to the $\nabla B$ drift in Fig. 1(b), while it has the same direction as the $\nabla B$ drift in Fig. 1(c).

In the following calculation, we restrict our attention to the $z=0$ plane, in which $\mathbf{B}_{x}$ vanishes and only $\mathbf{B}_{0}$ should be considered. As shown later, this restriction is justified as long as the curvature is small, that is,

$$
R_{c}^{2} \gg r_{L e}^{2} .
$$

It is the same magnetic field configuration as in the straight field case [Fig. 1(a)], but the curvature effects appear through a centrifugal force in the $+x$ or $-x$ directions [Figs. 1(b) or $1(\mathrm{c})]$.

We assume flute perturbations with $\mathbf{k} \cdot \mathbf{B}_{0}=0$, which has been shown to have the maximum growth rate by Gladd. ${ }^{12}$ Furthermore, it is assumed that

$$
k_{y}^{2} \gg k_{x}^{2} \gg \epsilon_{n}^{2}, \epsilon_{T}^{2}, \epsilon_{B}^{2},
$$

where $\epsilon_{n}=\partial \log n / \partial x, \epsilon_{T}=\partial \log T_{e} / \partial x$, and $\epsilon_{B}=\partial \log B_{0} / \partial x$. This implies that the wavelength in the $x$ direction is small in comparison with the gradient length scales and justifies the use of the local approximation. ${ }^{13}$ Moreover, in the present analysis, extraordinary-mode polarization is considered, with electric field perturbation $\delta \mathbf{E}(\mathbf{x}, t)=\delta E_{x}(\mathbf{x}, t) \hat{\mathbf{e}}_{x}$ $+\delta E_{y}(\mathbf{x}, t) \hat{\mathbf{e}}_{y}$, and magnetic field perturbation $\delta \mathbf{B}(\mathbf{x}, t)$ $=\delta B_{z}(\mathbf{x}, t) \hat{\mathbf{e}}_{z}$, where $\hat{\mathbf{e}}_{x}, \hat{\mathbf{e}}_{y}$, and $\hat{\mathbf{e}}_{z}$ are unit vectors in the $x$, $y$, and $z$ directions, respectively. 
We obtain the time-asymptotic results in the following way. First, we assume that all perturbations have the form

$$
\delta A(\mathbf{x}, t)=\delta \hat{A} \exp \left[i\left(k_{y} y-\omega t\right)\right], \quad \omega_{i}>0 .
$$

We then obtain the solution for $\omega_{i}>0$, and also for $\omega_{i} \leqslant 0$ by analytic continuation. Finally, we calculate a dispersion relation that indicates nontransient responses of normal modes.

\section{A. Ion contribution}

In the lower-hybrid-drift instability, ions are treated as unmagnetized:

$$
|\omega| \gg \Omega_{i}, \quad k_{y}^{2} r_{L i}^{2} \gg 1,
$$

where $r_{L i}=v_{i} / \Omega_{i}$ is the thermal ion Larmor radius. Here, we assume that the ion equilibrium distribution function depends on $w_{\perp}$ and $v_{\|}: f_{i 0}=f_{i 0}\left(w_{\perp}, v_{\|}\right)$, where we set $w_{\perp}=v_{x}^{2}$ $+\left(v_{y}-V_{i y}\right)^{2}$, and $V_{i y}$ is the ion fluid velocity. By Nakamura's method, ${ }^{10}$ we obtain the perturbed ion distribution function (see Appendix A)

$$
\begin{aligned}
\delta \hat{f}_{i}= & -\frac{2 i(+e)}{m_{i}} \frac{1}{\omega-k_{y} v_{y}}\left[v_{x}\left(1-\frac{k_{y} V_{i y}}{\omega}\right) \delta \hat{E}_{x}\right. \\
& \left.+\left(v_{y}-V_{i y}\right) \delta \hat{E}_{y}\right] \frac{\partial f_{i 0}}{\partial w_{\perp}},
\end{aligned}
$$

which is identical to the result by Davidson et al. ${ }^{7}$ [Eq. (33) therein].

\section{B. Electron contribution}

\section{1. $\nabla$ B drift}

We consider the lower-hybrid-drift instability with a straight ambient magnetic field shown in Fig. 1(a). Electrons are considered to be strongly magnetized:

$$
|\omega| \ll \Omega_{e}, \quad k_{y}^{2} r_{L e}^{2} \approx 1, \quad\left|r_{L e} \epsilon_{n}\right| \ll 1 .
$$

We set $f_{0 e}=f_{0 e}\left(\xi, w_{\perp}, v_{\|}\right)$, where $\xi=x-\left(v_{y}-V_{E}\right) / \Omega_{e}$, $w_{\perp}=v_{x}^{2}+\left(v_{y}-V_{E}\right)^{2}$, and $V_{E}=-E_{0} / B_{0}$ is the $\mathbf{E} \times \mathbf{B}$ drift velocity. The perturbed electron distribution function are then expressed as

$$
\begin{aligned}
\delta \hat{f}_{e}= & -\frac{(-e)}{m_{e}} \sum_{l, p} \frac{J_{p}(\mu) \exp \left[i(l-p)\left(\Omega_{e} t+\phi-\pi / 2\right)\right]}{k_{y}\left(V_{E}+V_{B}\right)+l \Omega_{e}-\omega} \\
& \times\left\{\frac{\sqrt{w_{\perp}}}{\omega}\left[-\frac{k_{y}}{\Omega_{e}} \frac{\partial f_{e 0}}{\partial \xi}+2\left(\omega-k_{y} V_{E}\right) \frac{\partial f_{e 0}}{\partial w_{\perp}}\right] J_{l}^{\prime}(\mu) \delta \hat{E}_{x}\right. \\
& \left.-i \frac{1}{k_{y}}\left[-\frac{k_{y}}{\Omega_{e}} \frac{\partial f_{e 0}}{\partial \xi}+2\left(l \Omega_{e}+k_{y} V_{B}\right) \frac{\partial f_{e 0}}{\partial w_{\perp}}\right] J_{l}(\mu) \delta \hat{E}_{y}\right\},
\end{aligned}
$$

which is equal to the result in Ref. 7 [Eq. (34) therein]. Here, we put $\phi=\tan ^{-1}\left[\left(v_{y}-V_{E}\right) / v_{x}\right]-\Omega_{e} t, V_{B}=-\epsilon_{B} w_{\perp} / 2 \Omega_{e}$ is the $\boldsymbol{\nabla} B$ drift velocity, $J_{l}(\mu)$ is the Bessel function of the first kind of order $l, J_{l}^{\prime}(\mu)=d J_{l}(\mu) / d \mu$ and $\mu=k_{y} \sqrt{w_{\perp}} / \Omega_{e}$ (a detailed derivation is given in Appendix A).

\section{2. $\nabla$ B drift + curvature drift}

Here, we add a curvature to the ambient magnetic field as shown in Figs. 1(b) or 1(c). The effect of the magnetic curvature is not only to cause a curvature drift of electrons, but also to modify a plane on which electrons gyrate. That is, the electron where the center of gyration is always on the $z$ $=0$ plane moves only on that plane, while the electron where the parallel velocity is zero and its center of gyration is above or below the $z=0$ plane has $z$-directional motion due to the gyration yielding the current in the $z$ direction. However, we can ignore the contribution of the electrons above and below the $z=0$ plane if the condition of $R_{c}^{2} \gg r_{L e}^{2}$ [Eq. (2)] is satisfied. This can be derived as follows: If an electron has a center of gyration on the $z=\delta z$, its displacement due to the gyration is about $\left(r_{L e} / R_{c}\right) \delta z$. If this becomes considerably small compared to $\delta z$, i.e., $R_{c}^{2} \gg r_{L e}^{2}$, contribution from such electrons can be ignored. Furthermore, we should note that the $z=0$ is not a special case. We can also consider a locally uniform finite $z$ region if we transform the coordinate such that the $z$ axis is tangential to the curved magnetic field.

We can then write the perturbed electron distribution function as

$$
\begin{aligned}
\delta \hat{f}_{e}= & -\frac{(-e)}{m_{e}} \sum_{l, p} \frac{J_{p}(\mu) \exp \left[i(l-p)\left(\Omega_{e} t+\phi-\pi / 2\right)\right]}{k_{y}\left(V_{E}+V_{B}+V_{c}\right)+l \Omega_{e}-\omega}\left\{\frac { \sqrt { w _ { \perp } } } { \omega } \left[-\frac{k_{y}}{\Omega_{e}} \frac{\partial f_{e 0}}{\partial \xi}+2\left(\omega-k_{y} V_{E}\right) \frac{\partial f_{e 0}}{\partial w_{\perp}}\right.\right. \\
& \left.-\frac{v_{\|}}{R_{c}} \frac{k_{y}}{\Omega_{e}} \sum_{n, q} \frac{I_{n}(\nu) I_{q}(\nu) i^{n+q} \exp \left[i(n-q)\left(\Omega_{e} t+\phi\right)\right]\left[k_{y}\left(V_{E}+V_{B}+V_{c}\right)+l \Omega_{e}-\omega\right]}{k_{y}\left(V_{E}+V_{B}+V_{c}\right)+(l+n) \Omega_{e}-\omega}\left(\frac{\partial f_{e 0}}{\partial v_{\|}}-2 v_{\|} \frac{\partial f_{e 0}}{\partial w_{\perp}}\right)\right] J_{l}^{\prime}(\mu) \delta \hat{E}_{x} \\
& -\frac{i}{k_{y}}\left[-\frac{k_{y}}{\Omega_{e}} \frac{\partial f_{e 0}}{\partial \xi}+2\left(l \Omega_{e}+k_{y} V_{B}+k_{y} V_{c}\right) \frac{\partial f_{e 0}}{\partial w_{\perp}}\right. \\
& \left.\left.-\frac{v_{\|}}{R_{c}} \frac{k_{y}}{\Omega_{e}} \sum_{n, q} \frac{I_{n}(\nu) I_{q}(\nu) i^{n+q} \exp \left[i(n-q)\left(\Omega_{e} t+\phi\right)\right]\left[k_{y}\left(V_{E}+V_{B}+V_{c}\right)+l \Omega_{e}-\omega\right]}{k_{y}\left(V_{E}+V_{B}+V_{c}\right)+(l+n) \Omega_{e}-\omega}\left(\frac{\partial f_{e 0}}{\partial v_{\|}}-2 v_{\|} \frac{\partial f_{e 0}}{\partial w_{\perp}}\right)\right] J_{l}(\mu) \delta \hat{E}_{y}\right\},
\end{aligned}
$$


where $V_{c}=\epsilon_{c} v_{\|}^{2} / \Omega_{e}$ is the curvature drift, $\epsilon_{c}=1 / R_{c}, I_{q}(\nu)$ is the modified Bessel function of the first kind of order $q$, and $\nu=\sqrt{w_{\perp}} / R_{c} \Omega_{e}$. Also see Appendix A for the derivation of Eq. (9).

\section{DISPERSION RELATION}

In this section, we give a concrete form of equilibrium distribution and calculate the perturbed charge and current density for ions and electrons from the perturbed distribution functions obtained in the preceding section. With the linearized Maxwell equations, we then derive the dispersion relations for the following three cases. The first case is when an ambient magnetic field is straight so that only the $\nabla B$ drift needs to be considered. The second case is when a virtual gravitational force is introduced to simulate the centrifugal force which acts if the magnetic field has a curvature. Note that the magnetic field is still straight even in the second case. Finally, we consider the case when the magnetic field is really curved and the curvature drift is properly treated.

\section{A. Equilibrium distribution}

For ions, we assume an equilibrium distribution function as a drifting Maxwellian,

$$
f_{i 0}\left(w_{\perp}, v_{\|}\right)=n\left(\frac{1}{\pi v_{i}^{2}}\right)^{3 / 2} \exp \left[-\frac{w_{\perp}+v_{\|}^{2}}{v_{i}^{2}}\right],
$$

where $w_{\perp}=v_{x}^{2}+\left(v_{y}-V_{i y}\right)^{2}$.

For electrons, we choose an equilibrium distribution as

$$
f_{e 0}\left(\xi, w_{\perp}, v_{\|}\right)=n(\xi)\left(\frac{1}{\pi v_{e}^{2}(\xi)}\right)^{3 / 2} \exp \left[-\frac{w_{\perp}+v_{\|}^{2}}{v_{e}^{2}(\xi)}\right]
$$

where $w_{\perp}=v_{x}^{2}+\left(v_{y}-V_{E}\right)^{2}, \xi=x-\left(v_{y}-V_{E}\right) / \Omega_{e}$.

For reference, we derive the dispersion relation when we simulate the effect of the magnetic curvature by the virtual gravitational force. Like before, ${ }^{4,13}$ the gravitational force $\mathbf{g}$ $=\left(2 T_{e} / m_{e} R_{c}\right) \hat{\mathbf{e}}_{x}$ is introduced, that is, the electron curvature drift is assumed to be an averaged value $\overline{\mathbf{V}}_{c}=g / \Omega_{e} \hat{\mathbf{e}}_{y}$. This assumption seen in previous works agrees with ours in that the curvature effects appear through a centrifugal force in the $x$ direction.

On the basis of this assumption, the local electron distribution function Eq. (11) is modified into

$f_{e 0}\left(\xi, w_{\perp}, v_{\|}\right)=n(\xi)\left(\frac{1}{\pi v_{e}^{2}(\xi)}\right)^{3 / 2} \exp \left[-\frac{w_{\perp}+v_{\|}^{2}}{v_{e}^{2}(\xi)}\right]$,

where $w_{\perp}=v_{x}^{2}+\left(v_{y}-V_{E}-\bar{V}_{c}\right)^{2}, \xi=x-\left(v_{y}-V_{E}-\bar{V}_{c}\right) / \Omega_{e}$, and $\bar{V}_{c}=\epsilon_{c} v_{e}^{2} / \Omega_{e}$ is the average curvature drift velocity. The distribution (12) indicates that all electrons are drifting at $\bar{V}_{c}$ in addition to $V_{E}$.

\section{B. Dispersion relation}

Calculating directly the perturbed charge and current density [ion contributions are from Eqs. (6) and (10); elec- tron contributions from Eqs. (8) and (11) for $\nabla B$ drift, from Eqs. (8) and (12) for $\nabla B$ drift + gravitational drift, and from Eqs. (9) and (11) for $\nabla B$ drift + curvature drift] and substitute them into the linearized Maxwell equations, we obtain the dispersion relation for perturbed electric field $\delta \hat{\mathbf{E}}$ :

$$
\begin{aligned}
{[1-} & \left.\frac{2 \omega_{p e}^{2}}{k_{y}^{2} v_{e}^{2}}\left(\Phi_{2}-C_{1}\right)+\frac{2 \omega_{p i}^{2}}{k_{y}^{2} v_{i}^{2}}\left[1+\zeta_{i} Z\left(\zeta_{i}\right)\right]\right] \\
& \times\left[1-\frac{c^{2} k_{y}^{2}}{\omega^{2}}-\frac{\omega_{p e}^{2}}{\omega^{2}} \Phi_{3}+\frac{\omega_{p i}^{2}}{\omega k_{y} v_{i}}\left(1-\frac{k_{y} V_{i y}}{\omega}\right) Z\left(\zeta_{i}\right)\right] \\
& -2\left(\frac{\omega_{p e}^{2}}{\omega k_{y} v_{e}}\right)^{2} \Phi_{1}\left(\Phi_{1}-C_{2}\right)=0,
\end{aligned}
$$

which determines the complex eigenfrequency $\omega=\omega_{r}$ $+i \omega_{i}$. Here,

$$
\begin{aligned}
& C_{1}=\frac{1}{v_{e}^{2}} \int_{0}^{\infty} d w_{\perp}\left[J_{0}(\mu)\right]^{2} \exp \left[-\frac{w_{\perp}}{v_{e}^{2}}\right], \\
& C_{2}=\frac{1}{v_{e}^{3}} \int_{0}^{\infty} d w_{\perp} \sqrt{w_{\perp}} J_{0}(\mu) J_{1}(\mu) \exp \left[-\frac{w_{\perp}}{v_{e}^{2}}\right],
\end{aligned}
$$

which are corrected [previously reported as $C_{1}=1$ and $C_{2}$ $=0$ (e.g., Ref. 7)]. $Z(\zeta)$ is the plasma dispersion function, ${ }^{14}$ which is introduced for analytic continuation to the all complex $\omega$ plane with $\zeta_{i}=\left(\omega-k_{y} V_{i y}\right) / k_{y} v_{i}$. The expressions of $\Phi_{j}(j=1,2,3)$ are given below in accordance with the situation.

\section{1. $\nabla$ B drift}

We find

$$
\begin{aligned}
\Phi_{j}= & \frac{1}{v_{e}^{2}} \int_{0}^{\infty} d w_{\perp} F_{j}\left(w_{\perp}\right) \exp \left[-\frac{w_{\perp}}{v_{e}^{2}}\right] \\
& \times\left[k_{y} V_{n}+k_{y} V_{T}\left(\frac{w_{\perp}}{v_{e}^{2}}-1\right)+k_{y} V_{E}-\omega\right] \\
& \times \lim _{k_{z} \rightarrow 0} \frac{1}{k_{z}} Z\left(\frac{\omega-k_{y} V_{E}-k_{y} V_{B}}{k_{z}}\right), \quad(j=1,2,3) .
\end{aligned}
$$

The functions $F_{j}\left(w_{\perp}\right)(j=1,2,3)$ appearing in the integrand are defined by

$$
\begin{aligned}
& F_{1}\left(w_{\perp}\right)=\frac{\sqrt{w_{\perp}}}{v_{e}} J_{0}(\mu) J_{1}(\mu), \\
& F_{2}\left(w_{\perp}\right)=\left[J_{0}(\mu)\right]^{2}, \\
& F_{3}\left(w_{\perp}\right)=\frac{w_{\perp}}{v_{e}^{2}}\left[J_{1}(\mu)\right]^{2} .
\end{aligned}
$$

Here, $V_{n}=-\epsilon_{n} v_{e}^{2} / 2 \Omega_{e}$ and $V_{T}=-\epsilon_{T} v_{e}^{2} / 2 \Omega_{e}$ are drifts associated with the density and temperature gradients, respectively. 


\section{2. $\nabla$ B drift + gravitational drift}

We find

$$
\begin{aligned}
\Phi_{j}= & \frac{1}{v_{e}^{2}} \int_{0}^{\infty} d w_{\perp} F_{j}\left(w_{\perp}\right) \exp \left[-\frac{w_{\perp}}{v_{e}^{2}}\right] \\
& \times\left[k_{y} V_{n}+k_{y} V_{T}\left(\frac{w_{\perp}}{v_{e}^{2}}-1\right)+k_{y} V_{E}+k_{y} \bar{V}_{c}-\omega\right] \\
& \times \lim _{k_{z} \rightarrow 0} \frac{1}{k_{z}} Z\left(\frac{\omega-k_{y} V_{E}-k_{y} V_{B}-k_{y} \bar{V}_{c}}{k_{z}}\right),
\end{aligned}
$$

where $F_{j}\left(w_{\perp}\right)(j=1,2,3)$ are given in Eqs. (17)-(19).

\section{3. $\nabla$ B drift + curvature drift}

We find

$$
\begin{aligned}
\Phi_{j}= & \frac{1}{v_{e}^{2}} \int_{0}^{\infty} d w_{\perp} F_{j}\left(w_{\perp}\right) \exp \left[-\frac{w_{\perp}}{v_{e}^{2}}\right] \\
& \times\left\{\frac{V_{T}}{\bar{V}_{c}}+\left[k_{y} V_{n}+k_{y} V_{T}\left(\frac{w_{\perp}}{v_{e}^{2}}-\frac{3}{2}\right)+k_{y} V_{E}-\omega\right.\right. \\
& \left.-\frac{V_{T}}{\bar{V}_{c}}\left(k_{y} V_{E}+k_{y} V_{B}-\omega\right)\right] \\
& \left.\times \frac{1}{k \bar{V}_{c}} G\left(\frac{\omega-k_{y} V_{E}-k_{y} V_{B}}{k_{y} \bar{V}_{c}}\right)\right\},
\end{aligned}
$$

where $F_{j}\left(w_{\perp}\right)(j=1,2,3)$ are also given in Eqs. (17)-(19), and we have introduced a new function named the altered plasma dispersion function $G(\zeta)$, which is defined by

$$
G(\zeta)=\frac{1}{\sqrt{\pi}} \int_{-\infty}^{\infty} d x \frac{\exp \left(-x^{2}\right)}{x^{2}-\zeta}
$$

for $0<\arg \zeta<2 \pi$ and by the analytic continuation of this for $\arg \zeta=0$ and $2 \pi \leqslant \arg \zeta<4 \pi$ (see Appendix C).

\section{NUMERICAL STUDIES ON DISPERSION RELATION}

We now solve Eq. (13) numerically for the complex eigenfrequency $\omega_{r}+i \omega_{i}$. We first rewrite the equations by the following eight dimensionless parameters: $V_{E} / v_{i}, r_{L i} \epsilon_{n}$, $r_{L i} \epsilon_{B}, \beta_{i}, T_{e} / T_{i}, r_{L i} \epsilon_{T}, V_{i y} / v_{i}$, and $R_{c} / L_{B}$, with normalizing $\omega_{r}$ and $\omega_{i}$ to $\omega_{L H}=\left(\Omega_{e} \Omega_{i}\right)^{1 / 2}\left(\simeq \Omega_{L H}\right.$ for $\omega_{p e}^{2} / \Omega_{e}^{2}$ $\gg 1$ ), and $k_{y}$ to $r_{L e}^{-1}$. Here, we put $L_{B}=1 / \epsilon_{B}$. These parameters are not independent, but have the following constraints. An equilibrium force balance on an ion fluid element in the $x$ direction requires $V_{i y}=V_{E}+V_{d i}$, where $V_{d i}$ $=\left(v_{i}^{2} / 2 \Omega_{i}\right) \partial \log \left(n T_{i}\right) / \partial x$ is the ion diamagnetic drift velocity. This equation is equivalent to

$$
\frac{V_{E}}{v_{i}}=\frac{V_{i y}}{v_{i}}-\frac{1}{2} r_{L i} \epsilon_{n},
$$

where we assume $\partial T_{i} / \partial x=0$. Moreover, the gradient properties are related self-consistently to other parameters through Ampère's law: $-\partial B_{0} / \partial x=\mu_{0} n e\left(V_{i y}-V_{y e}\right)$, that is,

\section{$\nabla \boldsymbol{B}$ Drift}
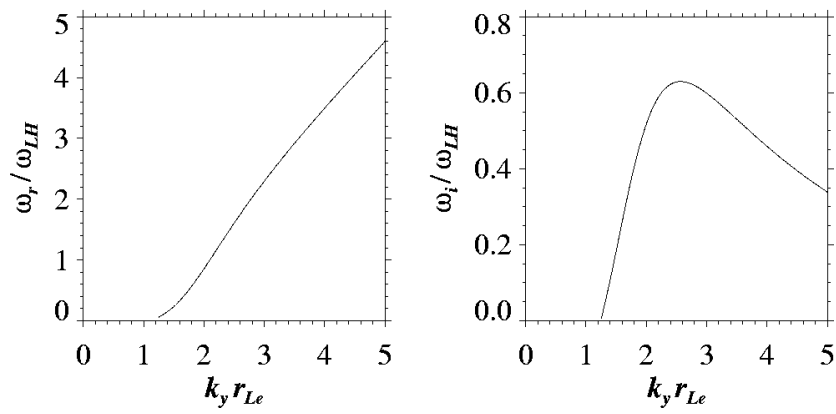

FIG. 2. Plots of $\omega_{r} / \omega_{L H}$ (left) and $\omega_{i} / \omega_{L H}$ (right) against $k_{y} r_{L e}$ obtained from numerical solutions to dispersion relations of the lower-hybrid-drift instability under a straight ambient magnetic field [Eqs. (13)-(19)]. The system parameters are $V_{E} / v_{i}=1, T_{e} / T_{i}=1, m_{e} / m_{i}=1 / 1836, \epsilon_{T}=0, \beta_{i}$ $=0.5, \omega_{p e}^{2} / \Omega_{e}^{2}=125$, and $V_{i y} / v_{i}=0$.

$$
\epsilon_{B}=-\frac{1}{2} \epsilon_{n}\left(\beta_{i}+\beta_{e}\right)-\frac{1}{2} \epsilon_{T} \beta_{e} .
$$

From Eqs. (23) and (24), we obtain

$$
r_{L i} \epsilon_{B}=\beta_{i}\left(1+\frac{T_{e}}{T_{i}}\right)\left(\frac{V_{E}}{v_{i}}-\frac{V_{i y}}{v_{i}}\right)-\frac{1}{2} \beta_{i} \frac{T_{e}}{T_{i}} r_{L i} \epsilon_{T} .
$$

In numerical studies, we typically specify $V_{E} / v_{i}, \beta_{i}$, $T_{e} / T_{i}, \quad r_{L i} \epsilon_{T}$, and $V_{i y} / v_{i}$, and then determine $r_{L i} \epsilon_{n}$ and $r_{L i} \epsilon_{B}$ consistently from Eqs. (23) and (25). Note that a variation in $\beta_{i}$ also involves a variation in other plasma parameters. Here, expressing $\beta_{i}=\left(\omega_{p e}^{2} / \Omega_{e}^{2}\right) \times\left(2 T_{i} / m_{e} c^{2}\right)$, we choose to vary $\beta_{i}$ by varying $\omega_{p e}^{2} / \Omega_{e}^{2}$ (with constant $T_{i}$ ). Following Ref. 7, we take $T_{i}=1.02 \mathrm{keV}$, so that $\beta_{i}=4$ $\times 10^{-3} \omega_{p e}^{2} / \Omega_{e}^{2}$. Since $R_{c} / L_{B}$ is independent of the parameters, we can substitute an arbitrary value for it. Moreover, we consider the case where the ions carry no current in the $y$ direction $\left(V_{i y}=0\right)$, so that the equilibrium electric field balances the ion pressure in the $x$ direction.

Figure 2 shows the dispersion relation for the lowerhybrid-drift instability under a straight magnetic field. We plot $\omega_{r} / \omega_{L H}$ (left) and $\omega_{i} / \omega_{L H}$ (right) against $k_{y} r_{L e}$ which satisfy Eqs. (13) -(19) for the choice of local plasma parameters: $\quad V_{E} / v_{i}=1, \quad T_{e} / T_{i}=1, \quad m_{e} / m_{i}=1 / 1836, \quad \epsilon_{T}=0$, $\omega_{p e}^{2} / \Omega_{e}^{2}=125$, and $\beta_{i}=0.5$, which are the same parameter selections as Ref. 7 .

The result is identical to that of previous works in that the real frequency $\omega_{r}$ is an increasing function of the wave number $k_{y}$. The growth rate $\omega_{i}$ achieves a maximum value $\omega_{i} \simeq 0.63 \omega_{L H}$ for $k_{y} \simeq 2.6 r_{L e}^{-1}$. This tendency can also be seen in Ref. 7 but the maximum value was $\omega_{i} \simeq 0.18 \omega_{L H}$ when $k_{y} \simeq 1.7 r_{L e}^{-1}$. This difference is brought by the corrected $C_{1}$ and $C_{2}$ [see Eqs. (14) and (15)].

Figure 3 shows $\omega_{r} / \omega_{L H}$ (left) and $\omega_{i} / \omega_{L H}$ (right) against $k_{y} r_{L e}$ including the gravitational drift [Eqs. (13)-(15) and (17)-(20)]. We choose the same parameters as in Fig. 2. In the upper two panels (a), we set the magnetic field positive curvature $R_{c} / L_{B}>0$, in which the curvature drift has the opposite sign to the $\nabla B$ drift. These panels show that both $\omega_{r}$ and $\omega_{i}$ increase with the curvature. Setting $R_{c} / L_{B}=4$ and 2 , the maximum growth rates increase to $1.0 \omega_{L H}$ and $1.6 \omega_{L H}$. 


\section{$\nabla \boldsymbol{B}$ Drift + Gravitational Drift}

(a) $R_{c} / L_{B}>0$
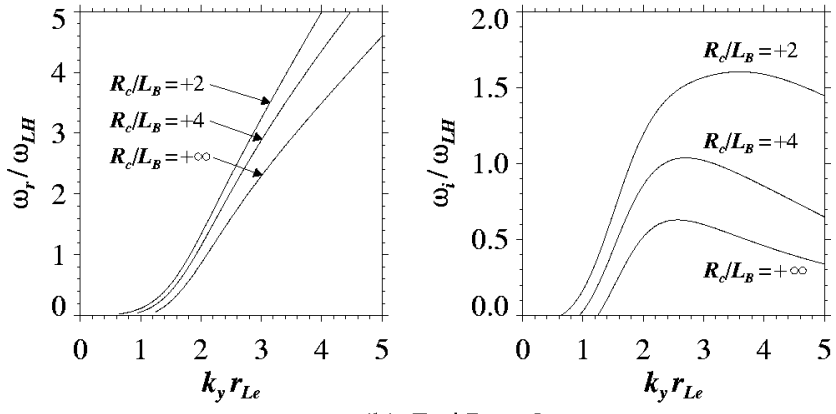

(b) $\boldsymbol{R}_{c} / \boldsymbol{L}_{B}<0$
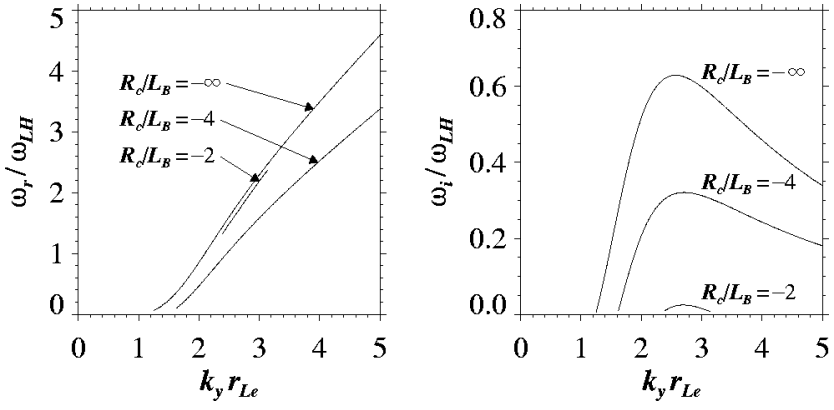

FIG. 3. Plots of $\omega_{r} / \omega_{L H}$ (left) and $\omega_{i} / \omega_{L H}$ (right) against $k_{y} r_{L e}$ obtained from numerical solutions to dispersion relations of the lower-hybrid-drift instability under a straight ambient magnetic field but including a virtual gravitational field for simulating the curvature effect [Eqs. (13)-(15) and (17)-(20)]. The top and bottom panels are (a) for $R_{c} / L_{B}=+\infty,+4,+2$ and (b) for $R_{c} / L_{B}=-\infty,-4,-2$. The other plasma parameters are the same as those in Fig. 2. Note that the range of $\omega_{i} / \omega_{L H}$ in panel (a) is taken wider than the other $\omega_{i} / \omega_{L H}$ against $k_{y} r_{L e}$ plots shown in Figs. 2 and 4 as well as in panel (b) of this figure.

On the other hand, we plot the dispersion for the magnetic field with the opposite curvature $\left(R_{c} / L_{B}<0\right)$ in Fig. 3(b), in which the curvature drift has the same direction as the $\nabla B$ drift. The growth rate $\omega_{i}$ decreases monotonically as the curvature becomes larger as shown in the right-hand panel of Fig. 3(b). The real frequency $\omega_{r}$, however, does not always change monotonically with respect to $R_{c} / L_{B}$. While $\omega_{r}$ decreases under the small curvature $\left(R_{c} / L_{B}=-\infty\right.$ and $-4)$, a larger curvature increases $\omega_{r}$ as seen in the graph for $R_{c} / L_{B}=-2$.

These characteristics are for the gravitational drift, in which a centrifugal force is simulated as a gravitational force. If we consider the curvature drift, we obtain different results. Figure 4 presents $\omega_{r} / \omega_{L H}$ (left) and $\omega_{i} / \omega_{L H}$ (right) against $k_{y} r_{L e}$ including the curvature drift [Eqs. (13)-(15), (17) $-(19)$, and (21)]. The parameters are also the same as in Fig. 2. Since the $G(\zeta)$ function appearing in Eq. (21) is a two-valued function with a single branch point of $\zeta=0$, we obtain the contributions of poles in both of two sheets. Here, we adopt the mode that is connected with the physically meaningful mode under an ambient magnetic field with no curvature.

First, we consider the case when an ambient magnetic field has a curvature such that $R_{c} / L_{B}>0$. The maximum $\omega_{i}$ becomes larger for a smaller curvature $\left(R_{c} / L_{B}=+\infty\right.$ and

\section{$\nabla \boldsymbol{B}$ Drift + Curvature Drift}

(a) $\boldsymbol{R}_{c} / \boldsymbol{L}_{B}>0$
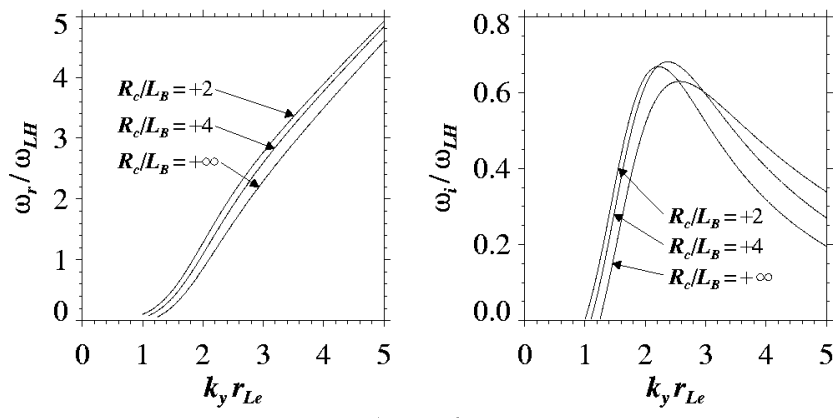

(b) $\boldsymbol{R}_{c} / \boldsymbol{L}_{B}<0$
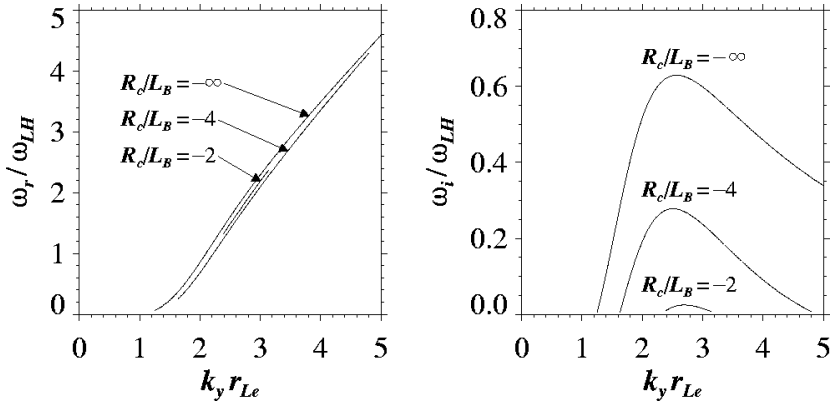

FIG. 4. Plots of $\omega_{r} / \omega_{L H}$ (left) and $\omega_{i} / \omega_{L H}$ (right) against $k_{y} r_{L e}$ obtained from numerical solutions to dispersion relations of the lower-hybrid-drift instability [Eqs. (13)-(15), (17)-(19), and (21)] under a curved ambient magnetic field. The top and bottom panels are (a) for $R_{c} / L_{B}=+\infty,+4,+2$ and (b) for $R_{c} / L_{B}=-\infty,-4,-2$. The other plasma parameters are the same as those in Figs. 2 and 3.

+4) as shown in the right-hand panel of Fig. 4(a). If the curve becomes more sharpened $\left(R_{c} / L_{B}=+2\right)$, however, the maximum $\omega_{i}$ gets smaller. This is in contrast to the tendency for the gravitational drift in that a larger curvature directly brings a larger growth rate. The values of maximum $\omega_{i}$ do not enlarge as in the gravitational drift approximation: $\omega_{i}$ $=0.68 \omega_{L H}$ and $0.67 \omega_{L H}$ for $R_{c} / L_{B}=+4$ and +2 .

The difference from the gravitational drift is also seen in the shape of $\omega_{i}-k_{y}$ curves [also see the right-hand panel of Fig. 3(a)]. The wave numbers giving the maximum growth rates shift to smaller values in accordance with the curvature, while they shift to larger values for the gravitational drift approximation. Furthermore, the curvature drift strongly reduces $\omega_{i}$ compared with the gravitational drift at larger $k_{y}$.

As can be seen in the gravitational drift, the real frequency $\omega_{r}$ gets larger when $R_{c} / L_{B}$ decreases, but the variation is small compared with the gravitational case.

On the other hand, when we set the magnetic field line on an opposite curvature $\left(R_{c} / L_{B}<0\right)$, we find similar results on $\omega_{r}$ and $\omega_{i}$ as in the gravitational drift approximation as shown in Fig. 4(b).

As we have seen in Fig. 4(a), $\omega_{i}$ has one peak for each $R_{c} / L_{B}$, whose value maximizes at $R_{c} / L_{B} \approx+4$. Searching for the $R_{c} / L_{B}$ that maximizes the peak value by the quasiNewton method, we find that $R_{c} / L_{B}=+3.595 \cdots$, in which the most effective resonances are expected to execute. In Fig. 5 , we demonstrate the effects of the $\nabla B$ drift and the curvature drift with a fixed $R_{c} / L_{B}=+3.595$. The four curves ap- 

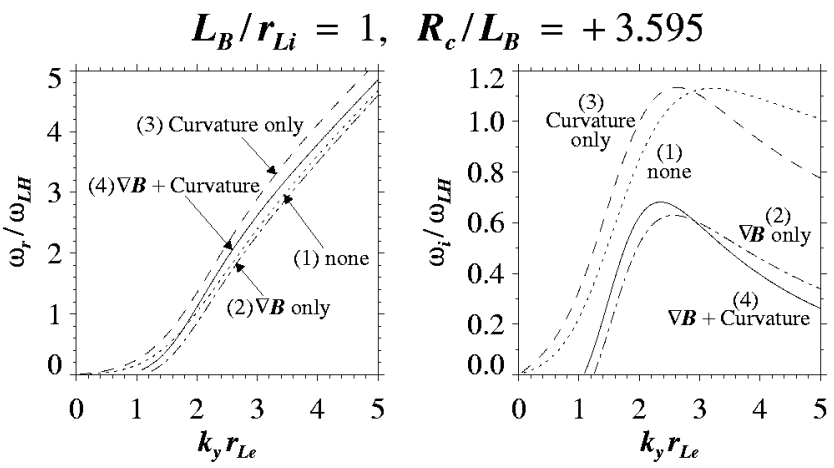

FIG. 5. Plots of $\omega_{r} / \omega_{L H}$ (left) and $\omega_{i} / \omega_{L H}$ (right) against $k_{y} r_{L e}$ : (1) the dotted line is without both $\boldsymbol{\nabla} B$ drift and curvature drift, (2) the dotteddashed line is with $\nabla B$ drift but without curvature drift, (3) the dashed line is with curvature drift but without $\nabla B$ drift, and (4) the solid line is with both drifts. The normalized radius of curvature $R_{c} / L_{B}$ is +3.595 , which gives the maximum value for the peak of the $\omega_{i} / \omega_{L H}-k_{y} r_{L e}$ curve for the parameter selections of Fig. 4

pearing in both panels are (1) neglecting both the $\nabla B$ and curvature drifts, (2) including the $\nabla B$ drift but neglecting the curvature drift, (3) including the curvature drift but neglecting the $\nabla B$ drift, and (4) including both drifts. These cases are executed arbitrarily by putting $V_{B}$ and $V_{c}$ equal to zero, which may be inconsistent with the definitions of $V_{B}$ and $V_{c}$ and Eq. (25), and this is done to understand the roles of these drifts.

The $\boldsymbol{\nabla} B$ drift decreases $\omega_{r}$, while the curvature drift increases $\omega_{r}$ as shown in the left-hand panel of Fig. 5 [see the curves of (1), (2), and (3)]. This tendency corresponds to a decrease of the phase velocity by the $\nabla B$ drift and an increase by the curvature drift. When both effects are concurrent [case (4)], the $\nabla B$ effect on $\omega_{r}$ becomes stronger than the curvature effect for a small $k_{y}\left(\lesssim 1.8 r_{L e}^{-1}\right)$ while the curvature effect on $\omega_{r}$ exceeds the $\nabla B$ effect for a large $k_{y}$ $\left(\gtrsim 1.8 r_{L e}^{-1}\right)$.

The growth rate decreases by about one-half by the $\nabla B$ effect shown in the right-hand panel of Fig. 5: the peak values are $\omega_{i}=1.13 \omega_{L H}$ in case (1), and $\omega_{i}=0.63 \omega_{L H}$ in case (2). On the other hand, the magnetic curvature shifts the peak to the smaller $k_{y}$, and decreases $\omega_{i}$ for the larger $k_{y}$ [case (3)]. Moreover, the magnetic curvature raises the peak value only when the $\nabla B$ effect is considered: the peak value of $\omega_{i}=0.63 \omega_{L H}$ in case (2) increases to $\omega_{i}=0.68 \omega_{L H}$ in case (4), while $\omega_{i}=1.13 \omega_{L H}$ in case (1) remains unchanged in case (3).

We directly compare the effects of the curvature drift and the gravitational drift for any radius of curvature in Fig. 6 . The other parameters are the same as in the previous plots. In the top-left panel of Fig. 6, we plot maximum growth rates normalized to $\omega_{L H}\left(\omega_{i, \mathrm{MAX}} / \omega_{L H}\right.$, maximized with respect to $k_{y}$ ) against $R_{c} / L_{B}$. When the ambient magnetic field has a curvature of $R_{c} / L_{B}>0, \omega_{i, \operatorname{MAX}}$ increases gradually up to $R_{c} / L_{B}=+3.595$ and then decreases rapidly by the curvature drift, while the gravitational drift brings a rapid and continuous increase of $\omega_{i, \operatorname{MAX}}$. On the other hand, when the magnetic field has the opposite curvature $\left(R_{c} / L_{B}<0\right)$, both drifts

\section{$\nabla \boldsymbol{B}$ Drift + Gravitational / Curvature Drift}
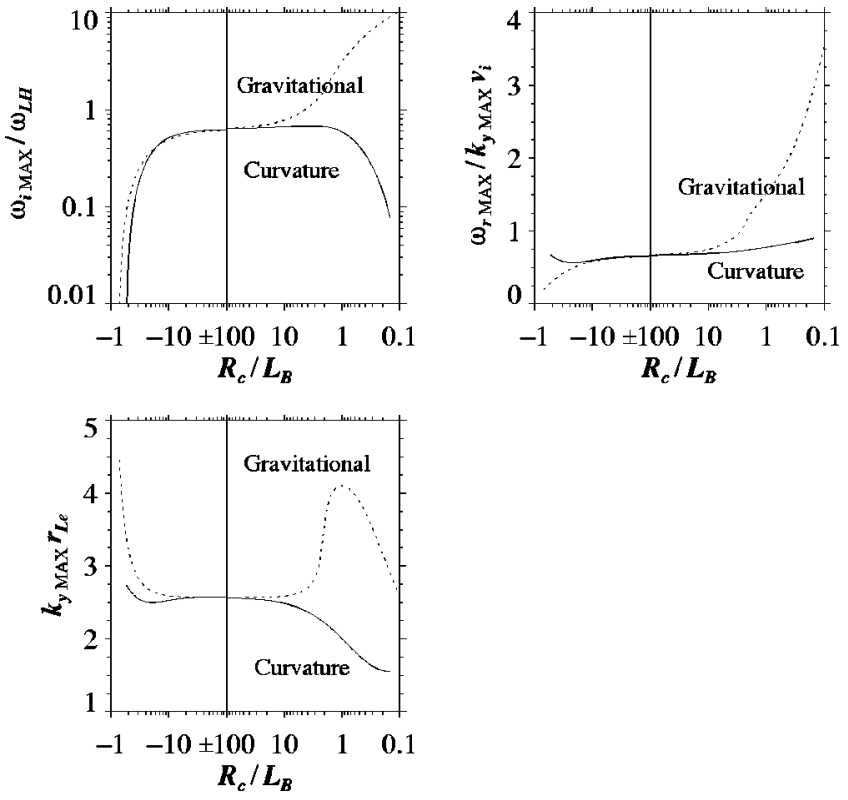

FIG. 6. Plots of normalized maximum growth rate $\left(\omega_{i, \mathrm{MAX}} / \omega_{L H}\right.$, in the top-left panel), normalized phase velocity at maximum growth $\left(\omega_{r, \operatorname{MAX}} / k_{y, \operatorname{MAX}} v_{i}\right.$, in the top-right panel), and normalized wave number at maximum growth $\left(k_{y, \operatorname{MAX}} r_{L e}\right.$, in the bottom panel) against $R_{c} / L_{B}$. The plasma parameters are the same as in Figs. 3 and 4.

have similar effects that reduce $\omega_{i, \operatorname{MAX}}$ gradually and then rapidly; the turning point is $R_{C} / L_{B} \simeq-10$.

The top-right panel of Fig. 6 shows phase velocities normalized to the ion thermal speed at maximum growth rates $\left(\omega_{r, \operatorname{MAX}} / k_{y, \operatorname{MAX}} v_{i}\right)$. In the case of the curvature drift, $\omega_{r, \operatorname{MAX}} / k_{y, \operatorname{MAX}}$ gradually increases when the magnetic field has a curvature of $R_{c} / L_{B}>0$ and gradually decreases when it has the opposite curvature. The value reached for a strongly curved magnetic field $\left(0.1<R_{c} / L_{B}<1\right)$ is found to be less than $1 v_{i}$, i.e., the value of $V_{E}$. The gravitational drift, however, does not bring such an upper limit: $\omega_{r, \operatorname{MAX}} / k_{y, \operatorname{MAX}}$ increases rapidly and reaches $3-4 v_{i}$ This is because the increasing gravitational drift practically enlarges $V_{E}$.

In the bottom panel of Fig. 6, we can see variations of normalized wave numbers at the maximum growth rate $\left(k_{y, \operatorname{MAX}} r_{L e}\right)$ against $R_{c} / L_{B}$. When the magnetic field has curvature, $k_{y, \operatorname{MAX}}$ decreases regardless of the curved magnetic field direction (except for the case of a strong negative curvature). On the other hand, $k_{y, \operatorname{MAX}}$ increases for the gravitational drift approximation, and a rapid increase of $k_{y, \operatorname{MAX}}$ can be seen at $R_{c} / L_{B} \simeq \pm 1$.

Finally, we examine the dependence on plasma beta in Fig. 7. In the top-left panel, we plot $\omega_{i, \mathrm{MAX}} / \omega_{L H}$ as a function of $R_{c} / L_{B}$, for several values of local $\beta_{i}$. The remaining parameters are the same as in Fig. 4. In the course of setting the magnetic field to have curvature such that $R_{c} / L_{B}=$ +100 to $+0.1, \omega_{i, \operatorname{MAX}}$ for each $\beta_{i}$ increases slowly to achieve the maximum at a certain value of $R_{c} / L_{B}$, then decreases rapidly. The values of $R_{c} / L_{B}$ giving the maximum $\omega_{i, \operatorname{MAX}}$ depend on $\beta_{i}$, and are $+10.7,+3.6,+1.18$, and +0.759 for $\beta_{i}=0.75,0.50,0.25$, and 0.10 , respectively. That is, the turning point of $\omega_{i, \operatorname{MAX}}$ from a slow increase to 

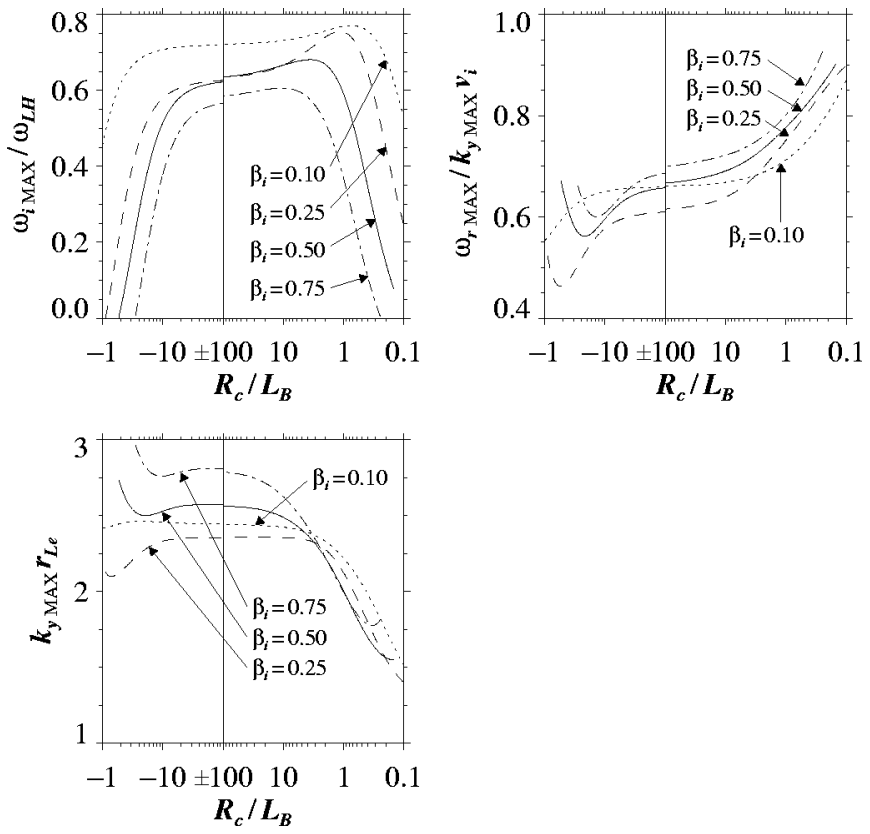

FIG. 7. Plots of normalized maximum growth rate $\left(\omega_{i, \mathrm{MAX}} / \omega_{L H}\right.$, top-left $)$, phase velocity at maximum growth $\left(\omega_{r, \operatorname{MAX}} / k_{y, \operatorname{MAX}} v_{i}\right.$, top-right), and wave number at maximum growth $\left(k_{y, \operatorname{MAX}} r_{L e}\right.$, bottom), against $R_{c} / L_{B}$ for $\left(\beta_{i}, \omega_{p e}^{2} / \Omega_{e}^{2}\right)=(0.1,25),(0.25,62.5),(0.50,125)$, and $(0.75,187.5)$. The other plasma parameters are the same as in Fig. 4.

a rapid decrease appears at a magnetic field with a smaller curvature when the ion beta is higher. In other words, the effect of a magnetic curvature becomes larger in higher beta environments. Furthermore, the peak values of $\omega_{i, \operatorname{MAX}}$ also depend on $\beta_{i}$. These values get larger with decreasing $\beta_{i}$ : $\omega_{i, \operatorname{MAX}}=0.771 \omega_{L H}, 0.756 \omega_{L H}, 0.681 \omega_{L H}$, and $0.606 \omega_{L H}$ for $\beta_{i}=0.75,0.50,0.25$, and 0.10 , respectively.

On the other hand, let us give the magnetic field the opposite curvature $R_{c} / L_{B}<0$. In this case, $\omega_{i, \operatorname{MAX}}$ decrease slowly and then rapidly for all $\beta_{i}$. The points where the slopes change depend on $\beta_{i}$ : they are $R_{c} / L_{B} \simeq-30,-20$, -10 , and -3 for $\beta_{i}=0.75,0.50,0.25$, and 0.10 , respectively. This finding also suggests that the magnetic curvature affects the maximum growth rate more significantly in a higher ion beta. On the whole, a lower ion beta provides a higher maximum growth rate, with the exception of the curves $\beta_{i}=0.50$ and $\beta_{i}=0.25$ around $R_{c} / L_{B} \approx 50$.

In the top-right panel of Fig. 7, we plot $\omega_{r, \operatorname{MAX}} / k_{y, \operatorname{MAX}} v_{i}$ for the same selections of local plasma parameters. These plots show that $\omega_{r, \operatorname{MAX}} / k_{y, \operatorname{MAX}}$ increases monotonically when an ambient magnetic field has a curvature of $R_{c} / L_{B}>0$, while they decrease under the opposite curvature $\left(R_{c} / L_{B}<0\right)$. If the magnetic field has a further negative curvature, phase velocities reach a minimum, and then begin to increase again, although the minimum does not appear in the displayed range of $-1>R_{c} / L_{B}>-100$ when $\beta_{i}=0.10$. The value of $\omega_{r, \operatorname{MAX}} / k_{y, \operatorname{MAX}}$ at a fixed $R_{c} / L_{B}$ is larger with increasing $\beta_{i}$ as seen for $\beta_{i}=0.25,0.50$, and 0.75 , but behaves in a different way for $\beta_{i}=0.10$.

In the bottom panel of Fig. 7, we plot $k_{y, \mathrm{MAX}} r_{L e}$ against $R_{c} / L_{B}$. When we curve the ambient magnetic field positively $\left(R_{c} / L_{B}>0\right), k_{y, \operatorname{MAX}}$ decreases for each $\beta_{i}$. The slope of the decreasing curve becomes sharper for a larger $\beta_{i}$, so that the order of $k_{y, \operatorname{MAX}}$ changes at $R_{c} / L_{B} \approx 3$ : a wave number is larger for a larger $\beta_{i}$ in $100>R_{c} / L_{B} \geq 3$, while all wave numbers are nearly the same for $R_{c} / L_{B} \leqslant 3$. With the opposite curvature $\left(R_{c} / L_{B}<0\right)$, we see slowly decreasing $k_{y, \operatorname{MAX}}$ against $R_{c} / L_{B}$, which have their minimum at $R_{C} / L_{B}=-9.5,-5.2$, and -1.4 for $\beta_{i}=0.75,0.50$, and 0.25 , respectively. As before, the curve for $\beta_{i}=0.10$ behaves in a slightly different way.

\section{CONCLUSION}

We have presented a linear study on the effects of magnetic curvature on the lower-hybrid-drift instability. In Sec. II we derived perturbed distribution functions by Nakamura's method, ${ }^{10}$ for an ambient magnetic field having curvature as well as for a straight magnetic field dealt with in past literature. ${ }^{1-3,5,7,11,12}$ In this derivation, we assumed that the curvature is small $\left(R_{c}^{2} \gg r_{L e}^{2}\right)$, so that the curvature effects appear only as a centrifugal force on electrons. The local dispersion relation for the lower-hybrid-drift instability was derived in Sec. III, including effects from both electron curvature drift and $\nabla B$ drift. For comparison, we also gave the dispersion relation with a virtual gravitational drift approximation of the curvature drift. In Sec. IV, local dispersion relations were solved numerically in the parameter regime of greatest interest for high-density pinches, i.e., $T_{e} \approx T_{i}$ and plasma betas on the order of unity. In calculations on the resonance of curvature drift, we introduced the altered dispersion function defined in Appendix C.

We summarize several important conclusions in the present analysis.

(1) When an ambient magnetic field has curvature so that the curvature drift is directed opposite to the $\nabla B$ drift, the maximum growth rate increases as long as the radius of curvature is larger than a certain value, while it decreases for a more curved magnetic field.

(2) In addition, when the magnetic field has the same curvature, the wave number giving the maximum growth decreases monotonically.

(3) On the other hand, when the magnetic field has the opposite curvature so that the curvature and $\nabla B$ drift have the same direction, the growth rate decreases monotonically.

Characteristics (1) and (2) cannot be revived by the gravitational drift approximation, though conclusion (3) is compatible with the result by the approximation. The difference between the curvature drift and the gravitational drift were summarized in Fig. 6.

(4) The growth rate is increased by the curvature drift coincident with the $\nabla B$ drift.

(5) The effect of the magnetic curvature becomes larger in high-beta plasmas. The curvature effect is, therefore, also important when dealing with the lower-hybrid-drift instability in the plasma sheet of the Earth's magnetotail, which is a high-beta region.

Several basic properties were clarified, but a substantial amount of work remains to be done to understand effects on this instability. For instance, we should explain the meaning of the deformation of dispersion due to magnetic curvature 
from a physical viewpoint. Furthermore, the change in the property in a low-beta plasma, which can be seen in Fig. 7, needs to be clarified. For such purposes, we will need detailed parameter studies or analytical surveys with some approximation limit.

On the basis of the three-dimensional particle simulations on a thin current sheet, Pritchett et al. ${ }^{15}$ reported that the normal magnetic field produces a mode having a wave vector of oblique angles to the main straight field and that lower-hybrid-drift modes are not excited due to the parallel component of the wave vector as pointed out by Gladd. ${ }^{12}$ The normal field is, of course, necessary to form an ambient magnetic field having a curvature, and can increase the growth rate of lower-hybrid-drift instability as presented in this paper. Moreover, the lower-hybrid-drift wave is not always reduced according to Ref. 11 . It then might also be worthwhile to include a component of a wave vector parallel to the ambient magnetic field in order to evaluate the effects of the magnetic curvature and the parallel component wave vector.

The resonance of curvature drift and wave would also modify the saturation level of the field fluctuations. ${ }^{16,17} \mathrm{~A}$ preliminary analysis in the $T_{e} \rightarrow 0$ limit shows that the saturation level is modified in the first order of $\mathbf{V}_{B}+\mathbf{V}_{c}$ and increases when $R_{c} / L_{B}>0$, which will cause an increase of anomalous resistivity. These studies will be addressed in a future paper.

\section{ACKNOWLEDGMENTS}

The author would like to thank Dr. T. K. Nakamura, Dr. I. Shinohara, and Dr. S. Machida for their valuable discussions.

\section{APPENDIX A: DERIVATION OF PERTURBED DISTRIBUTION FUNCTIONS}

Using the method by Nakamura, ${ }^{10}$ the perturbed distribution function $\delta f$ is written as

$$
\begin{aligned}
\delta f(\mathbf{q} ; t)= & -\left[\delta \Xi(\mathbf{q} ; t) \frac{\partial}{\partial \xi}+\delta W_{\perp}(\mathbf{q} ; t) \frac{\partial}{\partial w_{\perp}}+\delta V_{\|}(\mathbf{q} ; t) \frac{\partial}{\partial v_{\|}}\right] \\
& \times f_{0}\left(\xi, w_{\perp}, v_{\|}\right)+g\left(\xi, w_{\perp}, v_{\|}\right),
\end{aligned}
$$

where $\mathbf{q}=\left(\xi, y, w_{\perp}, v_{\|}, t\right)$ is a parameter set determining a particle orbit, $\xi=x-\left(v_{y}-V_{d}\right) / \Omega, w_{\perp}=v_{x}^{2}+\left(v_{y}-V_{d}\right)^{2}, v_{\|}$ $=v_{z}, V_{d}$ is the fluid velocity, $\Omega$ is the Larmor frequency, $f_{0}\left(\xi, w_{\perp}, v_{\|}\right)$is an unperturbed distribution function. The last term $g\left(\xi, w_{\perp}, v_{\|}\right)$is the initial perturbation, which is put equal to zero in the present derivation. We denote parameters to specify the phase space position by small letters and the corresponding physical quantities on the particle orbit by capital letters.

\section{Ion contribution}

The unperturbed velocity and position of an ion, which is regarded as moving at a constant velocity, are

$$
\begin{aligned}
& V_{x 0}\left(\mathbf{q} ; t^{\prime}\right)=v_{x}, \\
& V_{y 0}\left(\mathbf{q} ; t^{\prime}\right)=v_{y}, \\
& Y_{0}\left(\mathbf{q} ; t^{\prime}\right)=y+v_{y}\left(t^{\prime}-t\right) .
\end{aligned}
$$

Since we assume $f_{i 0}=f_{i 0}\left(w_{\perp}, v_{\|}\right)$, we need to obtain the perturbations $\delta W_{\perp}\left(\mathbf{q} ; t^{\prime}\right)$ and $\delta V_{\|}\left(\mathbf{q} ; t^{\prime}\right)$. First, from the $z$ component of the equation of motion:

$$
m_{i} \frac{d}{d t^{\prime}} \delta V_{\|}\left(\mathbf{q} ; t^{\prime}\right)=0,
$$

we obtain $\delta V_{\|}\left(\mathbf{q} ; t^{\prime}\right)=0$ under the initial condition $\delta V_{\|}\left(t^{\prime}\right.$ $=-\infty)=0$. We then consider the time variation of the kinetic energy in the moving coordinate with the velocity of $\mathbf{V}_{i y}=V_{i y} \hat{\mathbf{e}}_{y}$ relative to the rest frame. Since $\delta V_{\|}=0$,

$$
\begin{aligned}
\frac{d}{d t^{\prime}} \delta W_{\perp}\left(\mathbf{q} ; t^{\prime}\right)= & \frac{2(+e)}{m_{i}}\left[\left(1-\frac{k_{y} V_{i y}}{\omega}\right) \delta E_{x}\left(Y_{0}\left(\mathbf{q} ; t^{\prime}\right), t^{\prime}\right)\right. \\
& \times V_{x 0}\left(\mathbf{q} ; t^{\prime}\right)+\delta E_{y}\left(Y_{0}\left(\mathbf{q} ; t^{\prime}\right), t^{\prime}\right) \\
& \left.\times\left(V_{y 0}\left(\mathbf{q} ; t^{\prime}\right)-V_{i y}\right)\right]
\end{aligned}
$$

where Faraday's law $\delta B_{z}=\left(-k_{y} / \omega\right) \delta E_{x}$ was used to eliminate $\delta B_{z}$. Substituting the unperturbed orbit [Eqs. (A2)(A4)] into Eq. (A6) and integrating by $t^{\prime}$ from $-\infty$ to $t$, then we obtain

$$
\begin{aligned}
\delta \hat{W}_{\perp} & \exp \left[i\left(k_{y} y-\omega t\right)\right] \\
= & \frac{2 i(+e)}{m_{i}}\left[v_{x}\left(1-\frac{k_{y} V_{i y}}{\omega}\right) \delta \hat{E}_{x}+\left(v_{y}-V_{i y}\right) \delta \hat{E}_{y}\right] \\
& \times \frac{\exp \left[i\left(k_{y} y-\omega t\right)\right]}{\omega-k_{y} v_{y}} .
\end{aligned}
$$

Hence, the perturbed ion distribution function [Eq. (6)] is derived by calculating $\delta \hat{f}_{i}=-\delta \hat{W}_{\perp}\left(\partial f_{i 0} / \partial w_{\perp}\right)$.

\section{Electron contribution}

\section{a. $\nabla$ B drift}

Under a straight ambient magnetic field shown in Fig. 1(a), the unperturbed orbits are a gyro-motion plus guiding center drifts $(\mathbf{E} \times \mathbf{B}$ and $\boldsymbol{\nabla} B$ drifts):

$V_{x 0}\left(\mathbf{q} ; t^{\prime}\right)=\sqrt{w_{\perp}} \cos \left(\Omega_{e} t^{\prime}+\phi\right)$,

$V_{y 0}\left(\mathbf{q} ; t^{\prime}\right)=\sqrt{w_{\perp}} \sin \left(\Omega_{e} t^{\prime}+\phi\right)+V_{E}+V_{B}$,

$Y_{0}\left(\mathbf{q} ; t^{\prime}\right)=y+\left(V_{E}+V_{B}\right)\left(t^{\prime}-t\right)$

$$
-\frac{\sqrt{w_{\perp}}}{\Omega_{e}}\left[\cos \left(\Omega_{e} t^{\prime}+\phi\right)-\cos \left(\Omega_{e} t+\phi\right)\right] .
$$

When $f_{e 0}=f_{e 0}\left(\xi, w_{\perp}, v_{\|}\right)$, we need to obtain the perturbations $\delta \Xi\left(\mathbf{q} ; t^{\prime}\right), \delta W_{\perp}\left(\mathbf{q} ; t^{\prime}\right)$, and $\delta V_{\|}\left(\mathbf{q} ; t^{\prime}\right)$. In a similar manner as for ions, we find $\delta V_{\|}\left(\mathbf{q} ; t^{\prime}\right)=0$ by the force-free in the parallel direction. Also after a similar procedure as for ions, we obtain 


$$
\begin{aligned}
\delta \hat{W}_{\perp} \exp \left[i\left(k_{y} y-\omega t\right)\right] & \\
= & \frac{2(-e)}{m_{e}} \sum_{l, p} \frac{J_{p}(\mu) \exp \left[i(l-p)\left(\Omega_{e} t+\phi-\pi / 2\right)\right]}{k_{y}\left(V_{E}+V_{B}\right)+l \Omega_{e}-\omega} \\
& \times\left[\sqrt{w_{\perp}} J_{l}^{\prime}(\mu)\left(1-\frac{k_{y} V_{E}}{\omega}\right) \delta \hat{E}_{x}-i\left(\frac{l \Omega_{e}}{k_{y}}+V_{B}\right)\right. \\
& \left.\times J_{l}(\mu) \delta \hat{E}_{y}\right] \exp \left[i\left(k_{y} y-\omega t\right)\right]
\end{aligned}
$$

from the time variation of the kinetic energy in the moving frame with the velocity of $\mathbf{V}_{E}=V_{E} \hat{\mathbf{e}}_{y}$.

Next, we calculate the perturbed quantity $\delta \Xi\left(\mathbf{q} ; t^{\prime}\right)$. Utilizing the $y$ component of the equation of motion, we obtain

$$
\begin{aligned}
\frac{d}{d t^{\prime}} \delta \Xi\left(\mathbf{q} ; t^{\prime}\right)= & \frac{\delta E_{y}\left(Y_{0}\left(\mathbf{q} ; t^{\prime}\right), t^{\prime}\right)}{B_{0}\left(X_{0}\left(\mathbf{q} ; t^{\prime}\right)\right)}-V_{x 0}\left(\mathbf{q} ; t^{\prime}\right) \\
& \times \frac{\delta B_{z}\left(Y_{0}\left(\mathbf{q} ; t^{\prime}\right), t^{\prime}\right)}{B_{0}\left(X_{0}\left(\mathbf{q} ; t^{\prime}\right)\right)},
\end{aligned}
$$

where we evaluate the magnetic field value by $B_{0}\left(X_{0}\left(\mathbf{q} ; t^{\prime}\right)\right) \simeq B_{0}\left(x_{0}\right)=B_{0}$. Substituting the unperturbed orbit [Eqs. (A8)-(A10)] and integrating (A12) with respect to $t^{\prime}$, we obtain

$$
\begin{aligned}
\delta \hat{\Xi} \exp & {\left[i\left(k_{y} y-\omega t\right)\right] } \\
= & \frac{1}{B_{0}} \sum_{l, p} \frac{J_{p}(\mu) \exp \left[i(l-p)\left(\Omega_{e} t+\phi-\pi / 2\right)\right]}{k_{y}\left(V_{E}+V_{B}\right)+l \Omega_{e}-\omega} \\
& \times\left[\frac{k_{y} \sqrt{w_{\perp}}}{\omega} J_{l}^{\prime}(\mu) \delta \hat{E}_{x}-i J_{l}(\mu) \delta \hat{E}_{y}\right] \exp \left[i\left(k_{y} y-\omega t\right)\right] .
\end{aligned}
$$

From Eqs. (A11) and (A13), we can express the perturbed electron distribution function [Eq. (8)] by calculating $\delta \hat{f}_{e}=$ $-\left[\delta \hat{\Xi}(\partial / \partial \xi)+\delta \hat{W}_{\perp}\left(\partial / \partial w_{\perp}\right)\right] f_{e 0}$.

\section{b. $\nabla$ B drift + curvature drift}

When we add a curvature to the ambient magnetic field as shown in Fig. 1(b) or 1(c), the curvature drift participates in the unperturbed electron orbit

$V_{x 0}\left(\mathbf{q} ; t^{\prime}\right)=\sqrt{w_{\perp}} \cos \left(\Omega_{e} t^{\prime}+\phi\right)$,

$V_{y 0}\left(\mathbf{q} ; t^{\prime}\right)=\sqrt{w_{\perp}} \sin \left(\Omega_{e} t^{\prime}+\phi\right)+V_{E}+V_{B}+V_{c}$,

$V_{\| 0}\left(\mathbf{q} ; t^{\prime}\right)=v_{\|}$,

$$
\begin{aligned}
Y_{0}\left(\mathbf{q} ; t^{\prime}\right)= & y+\left(V_{E}+V_{B}+V_{c}\right)\left(t^{\prime}-t\right) \\
& -\frac{\sqrt{w_{\perp}}}{\Omega_{e}}\left[\cos \left(\Omega_{e} t^{\prime}+\phi\right)-\cos \left(\Omega_{e} t+\phi\right)\right] .
\end{aligned}
$$

In this case, we can also use the result of $\delta \Xi$ when only $\nabla B$ exists given by Eq. (A13).

As noted in Appendix B, $\delta V_{\|}$develops temporally governed by the differential equation

$$
\begin{aligned}
\frac{d}{d t^{\prime}} \delta V_{\|}\left(\mathbf{q} ; t^{\prime}\right)= & \frac{1}{R_{c}}\left[V_{x 0}\left(\mathbf{q} ; t^{\prime}\right) \delta V_{\|}\left(\mathbf{q} ; t^{\prime}\right)\right. \\
& \left.+V_{\| 0}\left(\mathbf{q} ; t^{\prime}\right) \delta V_{x}\left(\mathbf{q} ; t^{\prime}\right)\right] .
\end{aligned}
$$

From Eq. (A18), we obtain

$$
\begin{aligned}
\delta \hat{V}_{\|} \exp \left[i\left(k_{y} y-\omega t\right)\right]= & \frac{v_{\|}}{R_{c} B_{0}} \sum_{l, p, n, q} \frac{J_{p}(\mu) I_{n}(\nu) I_{q}(\nu) i^{n+q} \exp \left[i(l-p)\left(\Omega_{e} t+\phi-\pi / 2\right)\right] \exp \left[i(n-q)\left(\Omega_{e} t+\phi\right)\right]}{k_{y}\left(V_{E}+V_{B}+V_{c}\right)+(l+n) \Omega_{e}-\omega} \\
& \times\left[J_{l}^{\prime}(\mu) \frac{k_{y} \sqrt{w_{\perp}}}{\omega} \delta \hat{E}_{x}-i J_{l}(\mu) \delta \hat{E}_{y}\right] \exp \left[i\left(k_{y} y-\omega t\right)\right]
\end{aligned}
$$

under the initial condition $\delta V_{\|}\left(t^{\prime}=-\infty\right)=0$, where we used the unperturbed orbit (A14)-(A17) and the perturbed term

$$
\delta V_{x}\left(\mathbf{q} ; t^{\prime}\right)=\frac{1}{B_{0}}\left[\frac{k_{y} \sqrt{w_{\perp}}}{\omega} \cos \left(\Omega_{e} t^{\prime}+\phi\right) \delta E_{x}+\delta E_{y}\right] .
$$

$$
\begin{aligned}
\frac{d}{d t^{\prime}} \delta W_{\perp}\left(\mathbf{q} ; t^{\prime}\right)+2 \frac{d}{d t^{\prime}}\left[V_{\| 0}\left(\mathbf{q} ; t^{\prime}\right) \delta V_{\|}\left(\mathbf{q} ; t^{\prime}\right)\right] \\
=\frac{2(-e)}{m}\left[\left(1-\frac{k_{y} V_{E}}{\omega}\right) \delta E_{x}\left(Y_{0}\left(\mathbf{q} ; t^{\prime}\right), t^{\prime}\right) V_{x 0}\left(\mathbf{q} ; t^{\prime}\right)\right. \\
\left.\quad+\delta E_{y}\left(Y_{0}\left(\mathbf{q} ; t^{\prime}\right), t^{\prime}\right)\left(V_{y 0}\left(\mathbf{q} ; t^{\prime}\right)-V_{E}\right)\right]
\end{aligned}
$$

Finally, we can obtain $\delta W_{\perp}$ by considering the variation of the kinetic energy in the moving frame with the velocity of $\mathbf{V}_{E}=V_{E} \hat{\mathbf{e}}_{y}$ :
Substituting the unperturbed electron orbit (A14)-(A17) and integrating with respect to $t^{\prime}$ from $-\infty$ to $t$, we obtain 


$$
\begin{aligned}
\delta \hat{W}_{\perp} \exp \left[i\left(k_{y} y-\omega t\right)\right] & \frac{2(-e)}{m} \sum_{l, p} \frac{J_{p}(\mu) \exp \left[i(l-p)\left(\Omega_{e} t+\phi-\pi / 2\right)\right]}{k_{y}\left(V_{E}+V_{B}+V_{c}\right)+l \Omega_{e}-\omega} \\
& \times \exp \left[i\left(k_{y} y-\omega t\right)\right]\left[\sqrt{w_{\perp}} J_{l}^{\prime}(\mu)\left(1-\frac{k_{y} V_{E}}{\omega}\right) \delta \hat{E}_{x}\right. \\
& \left.-i\left(\frac{l \Omega_{e}}{k_{y}}+V_{B}+V_{c}\right) J_{l}(\mu) \delta \hat{E}_{y}\right] \\
& -2 v_{\|} \delta \hat{V}_{\|} \exp \left[i\left(k_{y} y-\omega t\right)\right] .
\end{aligned}
$$

From Eqs. (A13), (A19), and (A21), we can write down the perturbed electron distribution function [Eq. (9)] by $\delta \hat{f}_{e}$ $=-\left[\delta \hat{\Xi}(\partial / \partial \xi)+\delta \hat{W}_{\perp}\left(\partial / \partial w_{\perp}\right)+\delta \hat{V}_{\|}\left(\partial / \partial v_{\|}\right)\right] f_{e 0}$.

\section{APPENDIX B: TREATMENT OF MAGNETIC CURVATURE}

We take a cylindrical coordinate system $(r, \varphi, z)$, where $\hat{\mathbf{e}}_{\varphi}$ is the direction of the magnetic field, with the electric field perpendicular to it. The equations of motion of electrons are

$$
\begin{aligned}
& m_{e}\left(\frac{d v_{r}}{d t}-\frac{v_{\varphi}^{2}}{r}\right)=(-e)\left(E_{r}-B_{\varphi} v_{z}\right), \\
& m_{e}\left(\frac{d v_{\varphi}}{d t}+\frac{v_{\varphi} v_{r}}{r}\right)=0, \\
& m_{e} \frac{d v_{z}}{d t}=(-e)\left(E_{z}+B_{\varphi} v_{r}\right) .
\end{aligned}
$$

When we take the first perturbation of Eq. (B2), we obtain

$$
\frac{d \delta v_{\varphi}}{d t}=-\frac{1}{r}\left(v_{r 0} \delta v_{\varphi}+v_{\varphi 0} \delta v_{r}\right) .
$$

For the Cartesian coordinate system, we let $v_{r} \rightarrow-v_{x}, v_{\varphi}$ $\rightarrow v_{\|}, v_{z} \rightarrow v_{y}$, and $r \rightarrow R_{c}$.

\section{APPENDIX C: THE ALTERED PLASMA DISPERSION FUNCTION}

In the theory of linearized waves in a hot plasma with a zeroth order curved magnetic field, a certain function of complex argument, which we will call the altered plasma dispersion function, occurs when the unperturbed velocity distribution is taken to be Maxwellian. We define this function by

$$
G(\zeta)=\frac{1}{\sqrt{\pi}} \int_{-\infty}^{\infty} d x \frac{\exp \left(-x^{2}\right)}{x^{2}-\zeta}
$$

for $0<\arg \zeta<2 \pi$ and by the analytic continuation of this for $\arg \zeta=0$ and $2 \pi \leqslant \arg \zeta<4 \pi$. $G(\zeta)$ is a periodic function of period $4 \pi$ and has a Riemann surface consisting of two sheets with a single branch point of $\zeta=0$. The difference from the usual plasma dispersion function ${ }^{14}$ is the $x^{2}$ appearing in the denominator of the integrand instead of $x$. (a) $\arg \zeta=0$
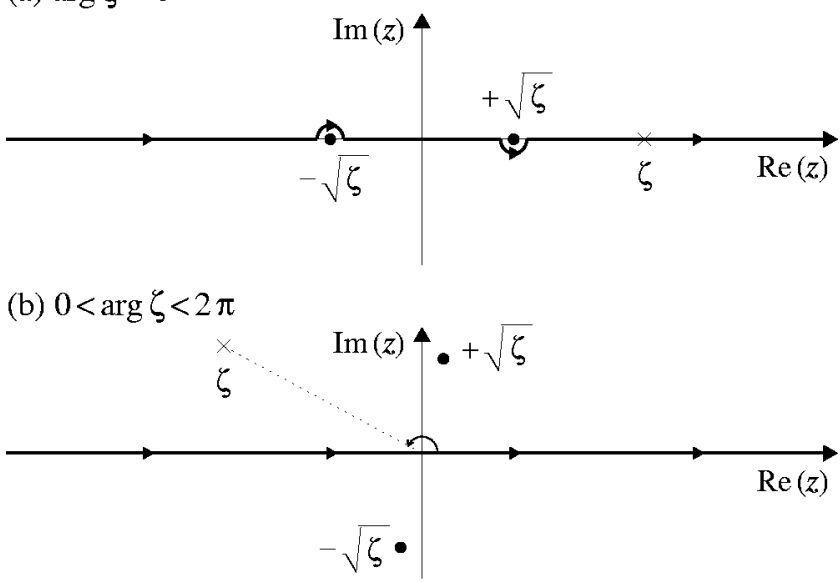

(c) $\arg \zeta=2 \pi$
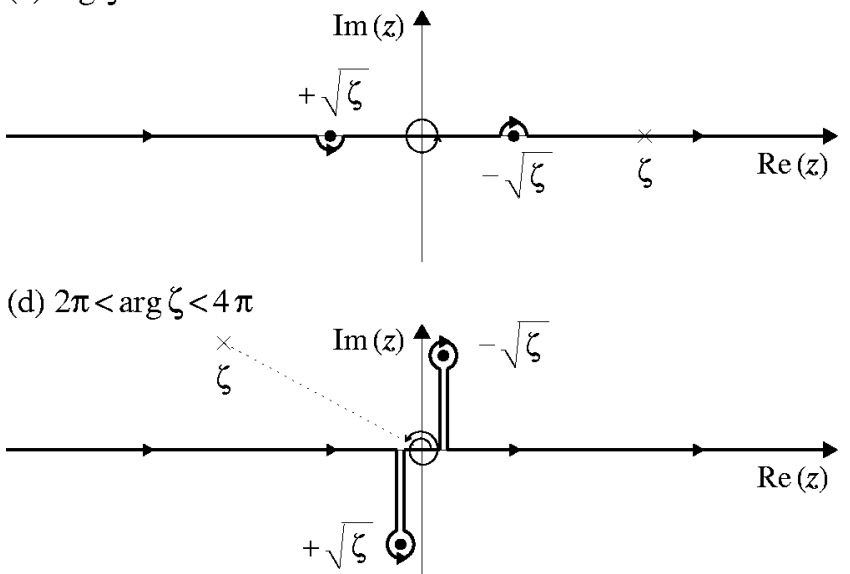

(e) $\arg \zeta=4 \pi$

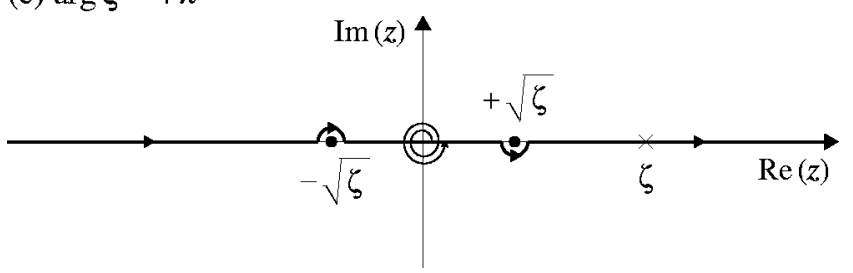

FIG. 8. Integral contour for evaluating $(1 / \sqrt{\pi}) \int_{-\infty}^{\infty} d x\left[\exp \left(-x^{2}\right) /\left(x^{2}-\zeta\right)\right]$ for five different values of $\arg \zeta$. This set of contours guarantees that the $x$ integral is a continuous function of $\zeta$.

We obtain the concrete expression of $G(\zeta)$ as follows. When we denote $\zeta=r \exp (i \theta)$, we find the two poles of $+\sqrt{\zeta}=\sqrt{r} \exp (i \theta / 2)$ and $-\sqrt{\zeta}=-(+\sqrt{\zeta})$. Taking account of the positions of these poles, we carry out integration such that the contour does not cross the poles.

Figure 8 shows integral contours for evaluating $G(\zeta)$ for five different values of $\arg \zeta$. For $0<\arg \zeta<2 \pi$ shown in Fig. 8(b), the contour is defined simply along the real $z$ axis, which is below the pole $+\sqrt{\zeta}$ and above the pole $-\sqrt{\zeta}$. At $\arg \zeta=0$, we deform the contour such that $+\sqrt{\zeta}$ and $-\sqrt{\zeta}$ are located above and below the contour, respectively. The deformed contour is shown in Fig. 8(a), and $G(\zeta)$ is defined by

$$
G(\zeta)=\frac{1}{\sqrt{\pi}} P \int_{-\infty}^{\infty} d x \frac{\exp \left(-x^{2}\right)}{x^{2}-\zeta}+i \sqrt{\pi} \frac{\exp (-\zeta)}{+\sqrt{\zeta}},
$$


where $P \int$ is the Cauchy principal value. When $\zeta$ makes one rotation from (a) through (b) and reaches (c), the contour should be deformed again such as (a). Different from (a), the contour passes under the pole at $\operatorname{Re}(z)<0$ and the pole at $\operatorname{Re}(z)>0$, but this is reasonable in view of the idea that the poles $+\sqrt{\zeta}$ and $-\sqrt{\zeta}$ are above and below the contour, respectively. Therefore, at $\arg \zeta=2 \pi, G(\zeta)$ is defined by

$$
G(\zeta)=\frac{1}{\sqrt{\pi}} P \int_{-\infty}^{\infty} d x \frac{\exp \left(-x^{2}\right)}{x^{2}-\zeta}+i \sqrt{\pi} \frac{\exp (-\zeta)}{+\sqrt{\zeta}} .
$$

When $\zeta$ rotates further and has the argument of $2 \pi<\arg \zeta$ $<4 \pi$, the contour is more deformed by a down-going $+\sqrt{\zeta}$ and an up-going $-\sqrt{\zeta}$ as shown in Fig. $8(\mathrm{~d}) . G(\zeta)$ is defined by

$$
G(\zeta)=\frac{1}{\sqrt{\pi}} \int_{-\infty}^{\infty} d x \frac{\exp \left(-x^{2}\right)}{x^{2}-\zeta}+2 i \sqrt{\pi} \frac{\exp (-\zeta)}{+\sqrt{\zeta}}
$$

for $2 \pi<\arg \zeta<4 \pi$. Finally, when $\zeta$ makes two rotations, the contour is shown in Fig. 8(e) which is equivalent to the $\arg \zeta=0$ case shown in Fig. 8(a).

When we calculate the integral appearing in the $G$ function, we deform the integral

$$
\frac{1}{\sqrt{\pi}} \int_{-\infty}^{\infty} d x \frac{\exp \left(-x^{2}\right)}{x^{2}-\zeta}=\frac{I(+\sqrt{\zeta})}{+\sqrt{\zeta}},
$$

where

$$
I(\zeta)=\frac{1}{\sqrt{\pi}} \int_{-\infty}^{\infty} d x \frac{\exp \left(-x^{2}\right)}{x-\zeta}=i \sqrt{\pi} w(\zeta)
$$

and $w(\zeta)$ is the complex error function. We then need to evaluate $w(\zeta)$, which is computed following Refs. 18 and 19 .

${ }^{1}$ N. A. Krall and P. C. Liewer, Phys. Rev. A 4, 2094 (1971).

${ }^{2}$ R. C. Davidson and N. T. Gladd, Phys. Fluids 18, 1327 (1975).

${ }^{3}$ J. D. Huba, N. T. Gladd, and K. Papadopoulos, J. Geophys. Res. 83, 5217 (1978).

${ }^{4}$ I. Shinohara, T. Nagai, M. Fujimoto, T. Terasawa, T. Mukai, K. Tsuruda, and T. Yamamoto, J. Geophys. Res. 103, 20365 (1998).

${ }^{5}$ J. D. Huba and C. S. Wu, Phys. Fluids 19, 988 (1976).

${ }^{6}$ J. D. Huba and J. F. Drake, Phys. Fluids 24, 1650 (1981).

${ }^{7}$ R. C. Davidson, N. T. Gladd, C. S. Wu, and J. D. Huba, Phys. Fluids 19, 1970 (1976).

${ }^{8}$ N. A. Krall and J. B. McBride, Phys. Fluids 19, 1970 (1976).

${ }^{9}$ J. D. Huba and J. F. Drake, Phys. Fluids 25, 1207 (1982).

${ }^{10}$ T. K. Nakamura, Phys. Plasmas 4, 3765 (1997).

${ }^{11}$ J. B. Hsia, S. M. Chiu, M. F. Hsia, R. L. Chou and C. S. Wu, Phys. Fluids 22, 1737 (1979)

${ }^{12}$ N. T. Gladd, Plasma Phys. 18, 27 (1976).

${ }^{13}$ N. A. Krall, in Advances in Plasma Physics 25, edited by A. Simon and W. B. Thompson (Wiley, New York, 1968), p. 153.

${ }^{14}$ B. D. Fried and S. D. Conte, The Plasma Dispersion Function (Academic, New York, 1961).

${ }^{15}$ P. L. Pritchett, F. V. Coroniti, and V. K. Decyk, J. Geophys. Res. 101, 27413 (1996).

${ }^{16}$ P. C. Liewer and R. C. Davidson, Nucl. Fusion 17, 85 (1977).

${ }^{17}$ R. C. Davidson, Phys. Fluids 21, 1375 (1978).

${ }^{18}$ W. Gautschi, SIAM (Soc. Ind. Appl. Math.) J. Numer. Anal. 7, 187 (1970).

${ }^{19}$ W. Gautschi, Commun. ACM 12, 635 (1969). 